\title{
MULTIPLE LEBESGUE INTEGRATION ON TIME SCALES
}

\author{
MARTIN BOHNER AND GUSEIN SH. GUSEINOV \\ Received 26 January 2006; Revised 17 April 2006; Accepted 18 April 2006
}

We study the process of multiple Lebesgue integration on time scales. The relationship of the Riemann and the Lebesgue multiple integrals is investigated.

Copyright (c) 2006 M. Bohner and G. Sh. Guseinov. This is an open access article distributed under the Creative Commons Attribution License, which permits unrestricted use, distribution, and reproduction in any medium, provided the original work is properly cited.

\section{Introduction}

Differential and integral calculus on time scales allows to develop a theory of dynamic equations in order to unify and extend the usual differential equations and difference equations. For single variable differential and integral calculus on time scales, we refer the reader to the textbooks $[4,5]$ and the references given therein. Multivariable calculus on time scales was developed by the authors [2, 3]. In [3], we presented the process of Riemann multiple delta (nabla and mixed types) integration on time scales. In the present paper, we introduce the definitions of Lebesgue multi-dimensional delta (nabla and mixed types) measures and integrals on time scales. A comparison of the Lebesgue multiple delta integral with the Riemann multiple delta integral is given.

Beside this introductory section, this paper consists of two sections. In Section 2, following [3], we give the Darboux definition of the Riemann multiple delta integral and present some needed facts connected to it. The main part of this paper is Section 3. There, a brief description of the Carathéodory construction of a Lebesgue measure in an abstract setting is given. Then the Lebesgue multi-dimensional delta measure on time scales is introduced and the Lebesgue delta measure of any single-point set is calculated. When we have a measure, integration theory is available according to the well-known general scheme of the Lebesgue integration process. Finally, we compare the Lebesgue multiple delta integral with the Riemann multiple delta integral. We indicate also a way to define, along with the Lebesgue multi-dimensional delta measure, the nabla and mixed types Lebesgue multi-dimensional measures on time scales. 


\section{Multiple Riemann integration}

In this section, following [3], we give the definition of the multiple Riemann integral on time scales over arbitrary bounded regions as we will compare in the next section the Riemann integral with the Lebesgue integral introduced therein. For convenience, we present the exposition for functions of two independent variables.

Let $\mathbb{T}_{1}$ and $\mathbb{T}_{2}$ be two time scales. For $i=1,2$, let $\sigma_{i}, \rho_{i}$, and $\Delta_{i}$ denote the forward jump operator, the backward jump operator, and the delta differentiation operator, respectively, on $\mathbb{T}_{i}$. Suppose $a<b$ are points in $\mathbb{T}_{1}, c<d$ are points in $\mathbb{T}_{2},[a, b)$ is the halfclosed bounded interval in $\mathbb{T}_{1}$, and $[c, d)$ is the half-closed bounded interval in $\mathbb{T}_{2}$. Let us introduce a "rectangle" (or "delta rectangle") in $\mathbb{T}_{1} \times \mathbb{T}_{2}$ by

$$
R=[a, b) \times[c, d)=\{(t, s): t \in[a, b), s \in[c, d)\} .
$$

First we define Riemann integrals over rectangles of the type given in (2.1). Let

$$
\begin{aligned}
& \left\{t_{0}, t_{1}, \ldots, t_{n}\right\} \subset[a, b], \quad \text { where } a=t_{0}<t_{1}<\cdots<t_{n}=b, \\
& \left\{s_{0}, s_{1}, \ldots, s_{k}\right\} \subset[c, d], \quad \text { where } c=s_{0}<s_{1}<\cdots<s_{k}=d .
\end{aligned}
$$

The numbers $n$ and $k$ may be arbitrary positive integers. We call the collection of intervals

$$
P_{1}=\left\{\left[t_{i-1}, t_{i}\right): 1 \leq i \leq n\right\}
$$

a $\Delta$-partition (or delta partition) of $[a, b)$ and denote the set of all $\Delta$-partitions of $[a, b)$ by $\mathscr{P}([a, b))$. Similarly, the collection of intervals

$$
P_{2}=\left\{\left[s_{j-1}, s_{j}\right): 1 \leq j \leq k\right\}
$$

is called a $\Delta$-partition of $[c, d)$ and the set of all $\Delta$-partitions of $[c, d)$ is denoted by $\mathscr{P}([c, d))$. Let us set

$$
R_{i j}=\left[t_{i-1}, t_{i}\right) \times\left[s_{j-1}, s_{j}\right), \quad \text { where } 1 \leq i \leq n, 1 \leq j \leq k .
$$

We call the collection

$$
P=\left\{R_{i j}: 1 \leq i \leq n, 1 \leq j \leq k\right\}
$$

a $\Delta$-partition of $R$, generated by the $\Delta$-partitions $P_{1}$ and $P_{2}$ of $[a, b)$ and $[c, d)$, respectively, and write $P=P_{1} \times P_{2}$. The rectangles $R_{i j}, 1 \leq i \leq n, 1 \leq j \leq k$, are called the subrectangles of the partition $P$. The set of all $\Delta$-partitions of $R$ is denoted by $\mathscr{P}(R)$.

Let $f: R \rightarrow \mathbb{R}$ be a bounded function. We set

$$
M=\sup \{f(t, s):(t, s) \in R\}, \quad m=\inf \{f(t, s):(t, s) \in R\}
$$

and for $1 \leq i \leq n, 1 \leq j \leq k$,

$$
M_{i j}=\sup \left\{f(t, s):(t, s) \in R_{i j}\right\}, \quad m_{i j}=\inf \left\{f(t, s):(t, s) \in R_{i j}\right\} .
$$


The upper Darboux $\Delta$-sum $U(f, P)$ and the lower Darboux $\Delta$-sum $L(f, P)$ of $f$ with respect to $P$ are defined by

$$
\begin{aligned}
& U(f, P)=\sum_{i=1}^{n} \sum_{j=1}^{k} M_{i j}\left(t_{i}-t_{i-1}\right)\left(s_{j}-s_{j-1}\right), \\
& L(f, P)=\sum_{i=1}^{n} \sum_{j=1}^{k} m_{i j}\left(t_{i}-t_{i-1}\right)\left(s_{j}-s_{j-1}\right) .
\end{aligned}
$$

Note that

$$
U(f, P) \leq \sum_{i=1}^{n} \sum_{j=1}^{k} M\left(t_{i}-t_{i-1}\right)\left(s_{j}-s_{j-1}\right)=M(b-a)(d-c)
$$

and likewise $L(f, P) \geq m(b-a)(d-c)$ so that

$$
m(b-a)(d-c) \leq L(f, P) \leq U(f, P) \leq M(b-a)(d-c) .
$$

The upper Darboux $\Delta$-integral $U(f)$ of $f$ over $R$ and the lower Darboux $\Delta$-integral $L(f)$ of $f$ over $R$ are defined by

$$
U(f)=\inf \{U(f, P): P \in \mathscr{P}(R)\}, \quad L(f)=\sup \{L(f, P): P \in \mathscr{P}(R)\} .
$$

In view of (2.11), $U(f)$ and $L(f)$ are finite real numbers. It can be shown that $L(f) \leq$ $U(f)$.

Definition 2.1. The function $f$ is called $\Delta$-integrable (or delta integrable) over $R$ provided $L(f)=U(f)$. In this case, $\iint_{R} f(t, s) \Delta_{1} t \Delta_{2} s$ is used to denote this common value. This integral is called the Riemann $\Delta$-integral.

We need the following auxiliary result. The proof can be found in [5, Lemma 5.7].

Lemma 2.2. For every $\delta>0$ there exists at least one partition $P_{1} \in \mathscr{P}([a, b))$ generated by a set

$$
\left\{t_{0}, t_{1}, \ldots, t_{n}\right\} \subset[a, b], \quad \text { where } a=t_{0}<t_{1}<\cdots<t_{n}=b,
$$

such that for each $i \in\{1,2, \ldots, n\}$ either

$$
t_{i}-t_{i-1} \leq \delta
$$

or

$$
t_{i}-t_{i-1}>\delta, \quad \rho_{1}\left(t_{i}\right)=t_{i-1} .
$$

Definition 2.3. The set of all $P_{1} \in \mathscr{P}([a, b))$ that possess the property indicated in Lemma 2.2 is denoted by $\mathscr{P}_{\delta}([a, b))$. Similarly, $\mathscr{P}_{\delta}([c, d))$ is defined. Further, the set of all $P \in \mathscr{P}(R)$ 
such that

$$
P=P_{1} \times P_{2}, \quad \text { where } P_{1} \in \mathscr{P}_{\delta}([a, b)), P_{2} \in \mathscr{P}_{\delta}([c, d))
$$

is denoted by $\mathscr{P}_{\delta}(R)$.

The following is a Cauchy criterion for Riemann delta integrability (see [3, Theorem 2.11]).

Theorem 2.4. A bounded function $f$ on $R$ is $\Delta$-integrable if and only if for each $\varepsilon>0$ there exists $\delta>0$ such that

$$
P \in \mathscr{P}_{\delta}(R) \text { implies } U(f, P)-L(f, P)<\varepsilon
$$

Remark 2.5. In the two-variable time scales case, four types of integrals can be defined:

(i) $\Delta \Delta$-integral over $[a, b) \times[c, d)$, which is introduced by using partitions consisting of subrectangles of the form $[\alpha, \beta) \times[\gamma, \delta)$;

(ii) $\nabla \nabla$-integral over $(a, b] \times(c, d]$, which is defined by using partitions consisting of subrectangles of the form $(\alpha, \beta] \times(\gamma, \delta]$;

(iii) $\Delta \nabla$-integral over $[a, b) \times(c, d]$, which is defined by using partitions consisting of subrectangles of the form $[\alpha, \beta) \times(\gamma, \delta]$;

(iv) $\nabla \Delta$-integral over $(a, b] \times[c, d)$, which is defined by using partitions consisting of subrectangles of the form $(\alpha, \beta] \times[\gamma, \delta)$.

For brevity the first integral we call simply a $\Delta$-integral, and in this paper we are dealing mainly with such $\Delta$-integrals.

For $i=1,2$ let us introduce the set $\mathbb{T}_{i}^{0}$ which is obtained from $\mathbb{T}_{i}$ by removing a possible finite maximal point of $\mathbb{T}_{i}$, that is, if $\mathbb{T}_{i}$ has a finite maximum $t^{*}$, then $\mathbb{T}_{i}^{0}=\mathbb{T}_{i} \backslash\left\{t^{*}\right\}$, otherwise $\mathbb{T}_{i}^{0}=\mathbb{T}_{i}$. Briefly we will write $\mathbb{T}_{i}^{0}=\mathbb{T}_{i} \backslash\left\{\max \mathbb{T}_{i}\right\}$. Evidently, for every point $t \in$ $\mathbb{T}_{i}^{0}$ there exists an interval of the form $[\alpha, \beta) \subset \mathbb{T}_{i}$ (with $\alpha, \beta \in \mathbb{T}_{i}$ and $\alpha<\beta$ ) that contains the point $t$.

Definition 2.6. Let $E \subset \mathbb{T}_{1}^{0} \times \mathbb{T}_{2}^{0}$ be a bounded set and let $f$ be a bounded function defined on the set $E$. Let $R=[a, b) \times[c, d) \subset \mathbb{T}_{1} \times \mathbb{T}_{2}$ be a rectangle containing $E$ (obviously such a rectangle $R$ exists) and define $F$ on $R$ as follows:

$$
F(t, s)= \begin{cases}f(t, s) & \text { if }(t, s) \in E \\ 0 & \text { if }(t, s) \in R \backslash E .\end{cases}
$$

Then $f$ is said to be Riemann $\Delta$-integrable over $E$ if $F$ is Riemann $\Delta$-integrable over $R$ in the sense of Definition 2.1, and

$$
\iint_{E} f(t, s) \Delta_{1} t \Delta_{2} s=\iint_{R} F(t, s) \Delta_{1} t \Delta_{2} s .
$$

If $E$ is an arbitrary bounded subset of $\mathbb{T}_{1}^{0} \times \mathbb{T}_{2}^{0}$, then even constant functions need not be Riemann $\Delta$-integrable over $E$. In connection with this we introduce the so-called Jordan $\Delta$-measurable subsets of $\mathbb{T}_{1}^{0} \times \mathbb{T}_{2}^{0}$. The definition makes use of the $\Delta$-boundary of 
a set $E \subset \mathbb{T}_{1} \times \mathbb{T}_{2}$. First, we recall the definition of the usual boundary of a set as given in $[3]$.

Definition 2.7. Let $E \subset \mathbb{T}_{1} \times \mathbb{T}_{2}$. A point $x=(t, s) \in \mathbb{T}_{1} \times \mathbb{T}_{2}$ is called a boundary point of $E$ if every open (two-dimensional) ball $B(x ; r)=\left\{y \in \mathbb{T}_{1} \times \mathbb{T}_{2}: d(x, y)<r\right\}$ of radius $r$ and center $x$ contains at least one point of $E$ and at least one point of $\left(\mathbb{T}_{1} \times \mathbb{T}_{2}\right) \backslash E$, where $d$ is the usual Euclidean distance. The set of all boundary points of $E$ is called the boundary of $E$ and is denoted by $\partial E$.

Definition 2.8. Let $E \subset \mathbb{T}_{1} \times \mathbb{T}_{2}$. A point $x=(t, s) \in \mathbb{T}_{1} \times \mathbb{T}_{2}$ is called a $\Delta$-boundary point of $E$ if $x \in \mathbb{T}_{1}^{0} \times \mathbb{T}_{2}^{0}$ and every rectangle of the form $\left[t, t^{\prime}\right) \times\left[s, s^{\prime}\right) \subset \mathbb{T}_{1} \times \mathbb{T}_{2}$ with $t^{\prime} \in$ $\mathbb{T}_{1}, t^{\prime}>t$, and $s^{\prime} \in \mathbb{T}_{2}, s^{\prime}>s$, contains at least one point of $E$ and at least one point of $\left(\mathbb{T}_{1} \times \mathbb{T}_{2}\right) \backslash E$. The set of all $\Delta$-boundary points of $E$ is called the $\Delta$-boundary of $E$, and it is denoted by $\partial_{\Delta} E$.

Definition 2.9. A point $\left(t^{0}, s^{0}\right) \in \mathbb{T}_{1}^{0} \times \mathbb{T}_{2}^{0}$ is called $\Delta$-dense if every rectangle of the form $V=\left[t^{0}, t\right) \times\left[s^{0}, s\right) \subset \mathbb{T}_{1} \times \mathbb{T}_{2}$ with $t \in \mathbb{T}_{1}, t>t^{0}$, and $s \in \mathbb{T}_{2}, s>s^{0}$, contains at least one point of $\mathbb{T}_{1} \times \mathbb{T}_{2}$ distinct from $\left(t^{0}, s^{0}\right)$. Otherwise the point $\left(t^{0}, s^{0}\right)$ is called $\Delta$-scattered.

Note that in the single variable case $\Delta$-dense points are precisely the right-dense points, and $\Delta$-scattered points are precisely the right-scattered points. Also, a point $\left(t^{0}, s^{0}\right) \in \mathbb{T}_{1}^{0} \times$ $\mathbb{T}_{2}^{0}$ is $\Delta$-dense if and only if at least one of $t^{0}$ and $s^{0}$ is right dense in $\mathbb{T}_{1}$ and $\mathbb{T}_{2}$, respectively.

Obviously, each $\Delta$-boundary point of $E$ is a boundary point of $E$, but the converse is not necessarily true. Also, each $\Delta$-boundary point of $E$ must belong to $\mathbb{T}_{1}^{0} \times \mathbb{T}_{2}^{0}$ and must be a $\Delta$-dense point in $\mathbb{T}_{1} \times \mathbb{T}_{2}$.

Example 2.10. We consider the following examples.

(i) For arbitrary time scales $\mathbb{T}_{1}$ and $\mathbb{T}_{2}$, the rectangle of the form $E=[a, b) \times[c, d) \subset$ $\mathbb{T}_{1} \times \mathbb{T}_{2}$, where $a, b \in \mathbb{T}_{1}, a<b$, and $c, d \in \mathbb{T}_{2}, c<d$, has no $\Delta$-boundary point, that is, $\partial_{\Delta} E=\varnothing$.

(ii) If $\mathbb{T}_{1}=\mathbb{T}_{2}=\mathbb{Z}$, then any set $E \subset \mathbb{Z} \times \mathbb{Z}$ has no boundary as well as no $\Delta$-boundary points.

(iii) Let $\mathbb{T}_{1}=\mathbb{T}_{2}=\mathbb{R}$ and $a, b, c, d \in \mathbb{R}$ with $a<b$ and $c<d$. Let us set

$$
E_{1}=[a, b) \times[c, d), \quad E_{2}=(a, b] \times(c, d], \quad E_{3}=[a, b] \times[c, d] .
$$

Then all three rectangles $E_{1}, E_{2}$, and $E_{3}$ have the boundary consisting of the union of all four sides of the rectangle. Moreover, $\partial_{\Delta} E_{1}$ is empty, $\partial_{\Delta} E_{2}$ consists of the union of all four sides of the rectangle $E_{2}$, and $\partial_{\Delta} E_{3}$ consists of the union of the right and upper sides of $E_{3}$.

(iv) Let $\mathbb{T}_{1}=\mathbb{T}_{2}=[0,1] \cup\{2\}$, where $[0,1]$ is the real number interval, and let $E=$ $[0,1) \times[0,1)$. Then the boundary $\partial E$ of $E$ consists of the union of the right and upper sides of the rectangle $E$ whereas $\partial_{\Delta} E=\varnothing$.

(v) Let $\mathbb{T}_{1}=\mathbb{T}_{2}=[0,1] \cup\{(n+1) / n: n \in \mathbb{N}\}$, where $[0,1]$ is the real number interval, and let $E=[0,1] \times[0,1]$. Then the boundary $\partial E$ as well as the $\Delta$-boundary $\partial_{\Delta} E$ of $E$ coincides with the union of the right and upper sides of $E$. 
Definition 2.11. Let $E \subset \mathbb{T}_{1}^{0} \times \mathbb{T}_{2}^{0}$ be a bounded set and let $\partial_{\Delta} E$ be its $\Delta$-boundary. Let $R=$ $[a, b) \times[c, d)$ be a rectangle in $\mathbb{T}_{1} \times \mathbb{T}_{2}$ such that $E \cup \partial_{\Delta} E \subset R$. Further, let $\mathscr{P}(R)$ denote the set of all $\Delta$-partitions of $R$ of type (2.5) and (2.6). For every $P \in \mathscr{P}(R)$ define $J_{*}(E, P)$ to be the sum of the areas (for a rectangle $V=[\alpha, \beta) \times[\gamma, \delta) \subset \mathbb{T}_{1} \times \mathbb{T}_{2}$, its "area" is the number $m(V)=(\beta-\alpha)(\delta-\gamma))$ of those subrectangles of $P$ which are entirely contained in $E$, and let $J^{*}(E, P)$ be the sum of the areas of those subrectangles of $P$ each of which contains at least one point of $E \cup \partial_{\Delta} E$. The numbers

$$
J_{*}(E)=\sup \left\{J_{*}(E, P): P \in \mathscr{P}(R)\right\}, \quad J^{*}(E)=\inf \left\{J^{*}(E, P): P \in \mathscr{P}(R)\right\}
$$

are called the (two-dimensional) inner and outer Jordan $\Delta$-measure of $E$, respectively. The set $E$ is said to be Jordan $\Delta$-measurable if $J_{*}(E)=J^{*}(E)$, in which case this common value is called the Jordan $\Delta$-measure of $E$, denoted by $J(E)$. The empty set is assumed to have Jordan $\Delta$-measure zero.

It is easy to verify that $0 \leq J_{*}(E) \leq J^{*}(E)$ always and that

$$
J^{*}(E)-J_{*}(E)=J^{*}\left(\partial_{\Delta} E\right)
$$

Hence $E$ is Jordan $\Delta$-measurable if and only if its $\Delta$-boundary $\partial_{\Delta} E$ has Jordan $\Delta$-measure zero.

Note that every rectangle $R=[a, b) \times[c, d) \subset \mathbb{T}_{1} \times \mathbb{T}_{2}$, where $a, b \in \mathbb{T}_{1}, a<b$, and $c, d \in \mathbb{T}_{2}, c<d$, is Jordan $\Delta$-measurable with Jordan $\Delta$-measure $J(R)=(b-a)(d-c)$. Indeed, it is easily seen that the $\Delta$-boundary of $R$ is empty, and therefore it has Jordan $\Delta$-measure zero.

For each point $x=(t, s) \in \mathbb{T}_{1}^{0} \times \mathbb{T}_{2}^{0}$, the single point set $\{x\}$ is Jordan $\Delta$-measurable, and its Jordan measure is given by

$$
J(\{x\})=\left(\sigma_{1}(t)-t\right)\left(\sigma_{2}(s)-s\right)=\mu_{1}(t) \mu_{2}(s) .
$$

Example 2.12. Let $\mathbb{T}_{1}=\mathbb{T}_{2}=\mathbb{R}\left(\right.$ or $\mathbb{T}_{1}=\mathbb{Z}$ and $\left.\mathbb{T}_{2}=\mathbb{R}\right)$, let $a<b$ be rational numbers in $\mathbb{T}_{1}$, and let $c<d$ be rational numbers in $\mathbb{T}_{2}$. Further, let $[a, b]$ be the interval in $\mathbb{T}_{1}$, let $[c, d]$ be the interval in $\mathbb{T}_{2}$, and let $E$ be the set of all points of $[a, b] \times[c, d]$ with rational coordinates. Then $E$ is not Jordan $\Delta$-measurable. The inner Jordan $\Delta$-measure of $E$ is 0 (there is no nonempty $\Delta$-rectangle entirely contained in $E$ ), while the outer Jordan $\Delta$ measure of $E$ is equal to $\left(\sigma_{1}(b)-a\right)(d-c)$. The $\Delta$-boundary of $E$ coincides with $E$ itself.

If $E \subset \mathbb{T}_{1}^{0} \times \mathbb{T}_{2}^{0}$ is any bounded Jordan $\Delta$-measurable set, then the integral $\iint_{E} 1 \Delta_{1} t \Delta_{2} s$ exists, and we have

$$
\iint_{E} 1 \Delta_{1} t \Delta_{2} s=J(E) .
$$


In the case $\mathbb{T}_{1}=\mathbb{T}_{2}=\mathbb{Z}$, for any bounded set $E \subset \mathbb{Z} \times \mathbb{Z}$ we have $\partial_{\Delta} E=0$, and therefore $E$ is Jordan $\Delta$-measurable. In this case, for any function $f: E \rightarrow \mathbb{R}$, we have

$$
\iint_{E} f(t, s) \Delta_{1} t \Delta_{2} s=\sum_{(t, s) \in E} f(t, s),
$$

and the Jordan $\Delta$-measure of $E$ coincides with the number of points of $E$.

Remark 2.13. Suppose that $\mathbb{T}_{1}$ has a finite maximum $\tau_{0}^{(1)}$. Since by definition $\sigma_{1}\left(\tau_{0}^{(1)}\right)=$ $\tau_{0}^{(1)}$, it is reasonable in view of (2.23) to assume that $J(\{x\})=0$ for any point $x$ of the form $x=\left(\tau_{0}^{(1)}, s\right)$, where $s \in \mathbb{T}_{2}$. Also, if $\mathbb{T}_{2}$ has a finite maximum $\tau_{0}^{(2)}$, then we can assume that $J(\{y\})=0$ for any point $y$ of the form $y=\left(t, \tau_{0}^{(2)}\right)$, where $t \in \mathbb{T}_{1}$.

\section{Multiple Lebesgue integration}

First, for the convenience of the reader, we briefly describe the Carathéodory construction of a Lebesgue measure in an abstract setting (see $[1,6-8]$ ). Let $X$ be an arbitrary set. A collection (family) $\mathscr{S}$ of subsets of $X$ is called a semiring if

(i) $\varnothing \in \mathscr{T}$;

(ii) $A, B \in \mathscr{Y}$, then $A \cap B \in \mathscr{Y}$;

(iii) $A, B \in \mathscr{Y}$ and $B \subset A$, then $A \backslash B$ can be represented as a finite union

$$
A \backslash B=\bigcup_{k=1}^{n} C_{k}
$$

of pairwise disjoint sets $C_{k} \in \mathscr{S}$.

A (set) function $m: \mathscr{Y} \rightarrow[0, \infty]$ whose domain is $\mathscr{S}$ and whose values belong to the extended real half-line $[0, \infty]$ is said to be a measure on $\mathscr{S}$ if

(i) $m(\varnothing)=0$;

(ii) $m$ is (finitely) additive in the sense that if $A \in \mathscr{S}$ such that $A=\cup_{i=1}^{n} A_{i}$, where $A_{1}, \ldots, A_{n} \in \mathscr{S}$ are pairwise disjoint, then

$$
m(A)=\sum_{i=1}^{n} m\left(A_{i}\right) .
$$

A measure $m$ with domain of definition $\mathscr{S}$ is said to be countably additive (or $\sigma$-additive) if, for every sequence $\left\{A_{i}\right\}$ of disjoint sets in $\mathscr{Y}$ whose union is also in $\mathscr{Y}$, we have

$$
m\left(\bigcup_{i=1}^{\infty} A_{i}\right)=\sum_{i=1}^{\infty} m\left(A_{i}\right) .
$$

Let $\mathscr{P}(X)$ be the collection of all subsets of $X, \mathscr{Y}$ a semiring of subsets of $X$, and $m: \mathscr{Y} \rightarrow$ $[0, \infty]$ a $\sigma$-additive measure of $\mathscr{Y}$. Define the set function

$$
m^{*}: \mathscr{P}(X) \longrightarrow[0, \infty]
$$


as follows. Let $E$ be any subset of $X$. If there exists at least one finite or countable system of sets $V_{i} \in \mathscr{Y}, i=1,2, \ldots$, such that $E \subset \cup_{i} V_{i}$, then we put

$$
m^{*}(E)=\inf \sum_{i} m\left(V_{i}\right)
$$

where the infimum is taken over all coverings of $E$ by a finite or countable system of sets $V_{i} \in \mathscr{Y}$. If there is no such covering of $E$, then we put $m^{*}(E)=\infty$. The set function $m^{*}$ defined above is called an outer measure on $\mathscr{P}(X)$ (or on $X$ ) generated by the pair $(\mathscr{Y}, m)$. The outer measure $m^{*}$ is defined for each $E \subset X$, however, it cannot be taken as a measure on $X$ because it is not additive in general. In order to guarantee the additivity of $m^{*}$ we must restrict $m^{*}$ to the collection of the so-called measurable subsets of $X$. A subset $A$ of $X$ is said to be $m^{*}$-measurable (or measurable with respect to $m^{*}$ ) if

$$
m^{*}(E)=m^{*}(E \cap A)+m^{*}\left(E \cap A^{C}\right), \quad E \subset X,
$$

where $A^{C}=X \backslash A$ denotes the complement of the set $A$. A fundamental fact of measure theory is that (see [6, Theorem 1.3.4]) the family $\mathbb{M}\left(m^{*}\right)$ of all $m^{*}$-measurable subsets of $X$ is a $\sigma$-algebra (i.e., $\mathbb{M}\left(m^{*}\right)$ contains $X$ and is closed under the formation of countable unions and of complements) and the restriction of $m^{*}$ to $\mathbb{M}\left(m^{*}\right)$, which we denote by $\mu$, is a $\sigma$-additive measure on $\mathbb{M}\left(m^{*}\right)$. We have $\mathscr{S} \subset \mathbb{M}\left(m^{*}\right)$ and $\mu(V)=m(V)$ for each $V \in \mathscr{Y}$. The measure $\mu$ is called the Carathéodory extension of the original measure $m$ defined on the semiring $\mathscr{Y}$. The measure $\mu$ obtained in this way is also called the Lebesgue measure on $X$ generated by the pair $(\mathscr{Y}, m)$. Note that the main difference between the Jordan and Lebesgue-Carathéodory constructions of measure of a set is that the Jordan construction makes use only of finite coverings of the set by some "elementary" sets whereas the Lebesgue-Carathéodory construction together with the finite coverings admits countable coverings as well. Due to this fact the notion of the Lebesgue measure generalizes the notion of the Jordan measure.

Passing now on to time scales, let $n \in \mathbb{N}$ be fixed. For each $i \in\{1, \ldots, n\}$, let $\mathbb{T}_{i}$ denote a time scale and let $\sigma_{i}, \rho_{i}$, and $\Delta_{i}$ denote the forward jump operator, the backward jump operator, and the delta differentiation operator on $\mathbb{T}_{i}$, respectively. Let us set

$$
\Lambda^{n}=\mathbb{T}_{1} \times \cdots \times \mathbb{T}_{n}=\left\{t=\left(t_{1}, \ldots, t_{n}\right): t_{i} \in \mathbb{T}_{i}, 1 \leq i \leq n\right\} .
$$

We call $\Lambda^{n}$ an $n$-dimensional time scale. The set $\Lambda^{n}$ is a complete metric space with the metric $d$ defined by

$$
d(t, s)=\left(\sum_{i=1}^{n}\left|t_{i}-s_{i}\right|^{2}\right)^{1 / 2} \quad \text { for } t, s \in \Lambda^{n} .
$$

Denote by $\mathscr{F}$ the collection of all rectangular parallelepipeds in $\Lambda^{n}$ of the form

$$
V=\left[a_{1}, b_{1}\right) \times \cdots \times\left[a_{n}, b_{n}\right)=\left\{t=\left(t_{1}, \ldots, t_{n}\right) \in \Lambda^{n}: a_{i} \leq t_{i}<b_{i}, 1 \leq i \leq n\right\}
$$

with $a=\left(a_{1}, \ldots, a_{n}\right), b=\left(b_{1}, \ldots, b_{n}\right) \in \Lambda^{n}$, and $a_{i} \leq b_{i}$ for all $1 \leq i \leq n$. If $a_{i}=b_{i}$ for some values of $i$, then $V$ is understood to be the empty set. We will call $V$ also an 
( $n$-dimensional) left-closed and right-open interval in $\Lambda^{n}$ and denote it by $[a, b)$. Let $m: \mathscr{F} \rightarrow[0, \infty)$ be the set function that assigns to each parallelepiped $V=[a, b)$ its volume:

$$
m(V)=\prod_{i=1}^{n}\left(b_{i}-a_{i}\right)
$$

Then it is not difficult to verify that $\mathscr{F}$ is a semiring of subsets of $\Lambda^{n}$ and $m$ is a $\sigma$-additive measure on $\mathscr{F}$. By $\mu_{\Delta}$ we denote the Carathéodory extension of the measure $m$ defined on the semiring $\mathscr{F}$ and call $\mu_{\Delta}$ the Lebesgue $\Delta$-measure on $\Lambda^{n}$. The $m^{*}$-measurable subsets of $\Lambda^{n}$ will be called $\Delta$-measurable sets and $m^{*}$-measurable functions will be called $\Delta$ measurable functions.

Theorem 3.1. Let $\mathbb{T}_{i}^{0}=\mathbb{T}_{i} \backslash\left\{\max \mathbb{\mathbb { T }}_{i}\right\}$. For each point

$$
t=\left(t_{1}, \ldots, t_{n}\right) \in \mathbb{T}_{1}^{0} \times \cdots \times \mathbb{T}_{n}^{0}
$$

the single-point set $\{t\}$ is $\Delta$-measurable, and its $\Delta$-measure is given by

$$
\mu_{\Delta}(\{t\})=\prod_{i=1}^{n}\left(\sigma_{i}\left(t_{i}\right)-t_{i}\right)=\prod_{i=1}^{n} \mu_{i}\left(t_{i}\right)
$$

Proof. If $t_{i}<\sigma_{i}\left(t_{i}\right)$ for all $1 \leq i \leq n$, then

$$
\{t\}=\left[t_{1}, \sigma_{1}\left(t_{1}\right)\right) \times \cdots \times\left[t_{n}, \sigma_{n}\left(t_{n}\right)\right) \in \mathscr{F} .
$$

Therefore $\{t\}$ is $\Delta$-measurable and

$$
\mu_{\Delta}(\{t\})=m\left(\left[t_{1}, \sigma_{1}\left(t_{1}\right)\right) \times \cdots \times\left[t_{n}, \sigma_{n}\left(t_{n}\right)\right)\right)=\prod_{i=1}^{n}\left(\sigma_{i}\left(t_{i}\right)-t_{i}\right),
$$

which is the desired result. Further consider the case when $t_{i}=\sigma_{i}\left(t_{i}\right)$ for some values of $i \in\{1, \ldots, n\}$ and $t_{i}<\sigma_{i}\left(t_{i}\right)$ for the other values of $i$. To illustrate the proof, suppose $t_{i}=\sigma_{i}\left(t_{i}\right)$ for $1 \leq i \leq n-1$ and $t_{n}<\sigma_{n}\left(t_{n}\right)$. In this case for each $i \in\{1, \ldots, n-1\}$ there exists a decreasing sequence $\left\{t_{i}^{(k)}\right\}_{k \in \mathbb{N}}$ of points of $\mathbb{T}_{i}$ such that $t_{i}^{(k)}>t_{i}$ and $t_{i}^{(k)} \rightarrow t_{i}$ as $k \rightarrow \infty$. Consider the parallelepipeds in $\Lambda^{n}$,

$$
V^{(k)}=\left[t_{1}, t_{1}^{(k)}\right) \times \cdots \times\left[t_{n-1}, t_{n-1}^{(k)}\right) \times\left[t_{n}, \sigma_{n}\left(t_{n}\right)\right) \quad \text { for } k \in \mathbb{N} \text {. }
$$

Then

$$
V^{(1)} \supset V^{(2)} \supset \cdots, \quad\{t\}=\bigcap_{k=1}^{\infty} V^{(k)}
$$

Hence $\{t\}$ is $\Delta$-measurable as a countable intersection of $\Delta$-measurable sets, and by the 
continuity property of the $\sigma$-additive measure $\mu_{\Delta}$, we have

$$
\mu_{\Delta}(\{t\})=\lim _{k \rightarrow \infty} \mu_{\Delta}\left(V^{(k)}\right)=\lim _{k \rightarrow \infty}\left(t_{1}^{(k)}-t_{1}\right) \cdots\left(t_{n-1}^{(k)}-t_{n-1}\right)\left(\sigma_{n}\left(t_{n}\right)-t_{n}\right)=0,
$$

and so (3.12) holds in this case as well.

Example 3.2. In the case $\mathbb{T}_{1}=\cdots=\mathbb{T}_{n}=\mathbb{R}$, the measure $\mu_{\Delta}$ coincides with the ordinary Lebesgue measure on $\mathbb{R}^{n}$. In the case $\mathbb{T}_{1}=\cdots=\mathbb{T}_{n}=\mathbb{Z}$, for any $E \subset \mathbb{Z}^{n}, \mu_{\Delta}(E)$ coincides with the number of points of the set $E$.

Example 3.3. The set $E$ given above in Example 2.12 is Lebesgue $\Delta$-measurable and its (two-dimensional) Lebesgue $\Delta$-measure is equal to zero.

Having the $\sigma$-additive measure $\mu_{\Delta}$ on $\Lambda^{n}$, we possess the corresponding integration theory for functions $f: E \subset \Lambda^{n} \rightarrow \mathbb{R}$, according to the general Lebesgue integration theory (see, e.g., [6]). The Lebesgue integral associated with the measure $\mu_{\Delta}$ on $\Lambda^{n}$ is called the Lebesgue $\Delta$-integral. For a $\Delta$-measurable set $E \subset \Lambda^{n}$ and a $\Delta$-measurable function $f: E \rightarrow$ $\mathbb{R}$, the corresponding $\Delta$-integral of $f$ over $E$ will be denoted by

$$
\int_{E} f\left(t_{1}, \ldots, t_{n}\right) \Delta_{1} t_{1} \cdots \Delta_{n} t_{n}, \quad \int_{E} f(t) \Delta t, \quad \text { or } \quad \int_{E} f d \mu_{\Delta} .
$$

So all theorems of the general Lebesgue integration theory, including the Lebesgue dominated convergence theorem, hold also for Lebesgue $\Delta$-integrals on $\Lambda^{n}$. Finally, we compare the Lebesgue $\Delta$-integral with the Riemann $\Delta$-integral.

Theorem 3.4. Let $V=[a, b)$ be a rectangular parallelepiped in $\Lambda^{n}$ of the form (3.9) and let $f$ be a bounded real-valued function on $V$. If $f$ is Riemann $\Delta$-integrable over $V$, then $f$ is Lebesgue $\Delta$-integrable over $V$, and

$$
R \int_{V} f(t) \Delta t=L \int_{V} f(t) \Delta t
$$

where $R$ and L indicate the Riemann and Lebesgue $\Delta$-integrals, respectively.

Proof. Suppose that $f$ is Riemann $\Delta$-integrable over $V=[a, b)$. Then, by Theorem 2.4, for each $k \in \mathbb{N}$ we can choose $\delta_{k}>0$ (with $\delta_{k} \rightarrow 0$ as $k \rightarrow \infty$ ) and a $\Delta$-partition $P^{(k)}=$ $\left\{R_{i}^{(k)}\right\}_{i=1}^{N(k)}$ of $V$ such that $P^{(k)} \in \mathscr{P}_{\delta_{k}}(V)$ and $U\left(f, P^{(k)}\right)-L\left(f, P^{(k)}\right)<1 / k$. Hence

$$
\lim _{k \rightarrow \infty} L\left(f, P^{(k)}\right)=\lim _{k \rightarrow \infty} U\left(f, P^{(k)}\right)=R \int_{V} f(t) \Delta t .
$$

By replacing the partitions $P^{(k)}$ with finer partitions if necessary, we can assume that for each $k \in \mathbb{N}$ the partition $P^{(k+1)}$ is a refinement of the partition $P^{(k)}$. Let us set

$$
m_{i}^{(k)}=\inf \left\{f(t): t \in R_{i}^{(k)}\right\}, \quad M_{i}^{(k)}=\sup \left\{f(t): t \in R_{i}^{(k)}\right\}
$$

for $i=1,2, \ldots, N(k)$, and define sequences $\left\{\varphi_{k}\right\}_{k \in \mathbb{N}}$ and $\left\{\phi_{k}\right\}_{k \in \mathbb{N}}$ of functions on $V$ by letting

$$
\varphi_{k}(t) \equiv m_{i}^{(k)}, \quad \phi_{k}(t) \equiv M_{i}^{(k)} \quad \text { for } t \in R_{i}^{(k)}, 1 \leq i \leq N(k)
$$


Then $\left\{\varphi_{k}\right\}$ is a nondecreasing and $\left\{\phi_{k}\right\}$ is a nonincreasing sequence of simple $\Delta$-measurable functions. For each $k \in \mathbb{N}$, we have

$$
\begin{gathered}
\varphi_{k} \leq \varphi_{k+1}, \quad \phi_{k} \geq \phi_{k+1}, \\
\varphi_{k} \leq f \leq \phi_{k} \\
L \int_{V} \varphi_{k}(t) \Delta t=L\left(f, P^{(k)}\right), \quad L \int_{V} \phi_{k}(t) \Delta t=U\left(f, P^{(k)}\right) .
\end{gathered}
$$

Since $f$ is bounded, the sequences $\left\{\varphi_{k}\right\}$ and $\left\{\phi_{k}\right\}$ are bounded by (3.21) and (3.22). Additionally taking the monotonicity (3.23) into account, we conclude that the limit functions

$$
\varphi(t)=\lim _{k \rightarrow \infty} \varphi_{k}(t), \quad \phi(t)=\lim _{k \rightarrow \infty} \phi_{k}(t) \quad \text { for } t \in V
$$

exist and that

$$
\varphi(t) \leq f(t) \leq \phi(t), \quad t \in V
$$

The functions $\varphi$ and $\phi$ are $\Delta$-measurable as limits (3.26) of the $\Delta$-measurable functions $\varphi_{k}$ and $\phi_{k}$, respectively. Lebesgue's dominated convergence theorem implies that $\varphi$ and $\phi$ are Lebesgue $\Delta$-integrable over $V$ and

$$
\lim _{k \rightarrow \infty} L \int_{V} \varphi_{k}(t) \Delta t=L \int_{V} \varphi(t) \Delta t, \quad \lim _{k \rightarrow \infty} L \int_{V} \phi_{k}(t) \Delta t=L \int_{V} \phi(t) \Delta t .
$$

Therefore passing on to the limit in (3.25) as $k \rightarrow \infty$ and taking (3.20) into account, we obtain

$$
L \int_{V} \varphi(t) \Delta t=L \int_{V} \phi(t) \Delta t=R \int_{V} f(t) \Delta t
$$

From (3.29) we find

$$
L \int_{V}[\phi(t)-\varphi(t)] \Delta t=0
$$

Since in addition $\phi(t)-\varphi(t) \geq 0$ for all $t \in V,(3.30)$ implies

$$
\varphi(t)=\phi(t) \quad \Delta \text {-almost everywhere on } V .
$$

So (3.27) and (3.31) show that $f(t)=\varphi(t) \Delta$-almost everywhere on $V$. It follows that $f$ is Lebesgue $\Delta$-integrable together with $\varphi$ and that the Lebesgue $\Delta$-integrals of $f$ and $\varphi$ over $V$ coincide. Now from (3.29) we get that the Lebesgue $\Delta$-integral of $f$ over $V$ coincides with the Riemann $\Delta$-integral of $f$ over $V$.

Remark 3.5. Let $E$ be a bounded Jordan $\Delta$-measurable set in $\mathbb{T}_{1}^{0} \times \cdots \times \mathbb{T}_{n}^{0}$ and $f: E \rightarrow \mathbb{R}$ a bounded function. Using Definition 2.6 and Theorem 3.4 we get that if $f$ is Riemann $\Delta$ integrable over $E$, then $f$ is Lebesgue $\Delta$-integrable over $E$, and the values of the integrals coincide. 
Remark 3.6. We can define Lebesgue nabla measure (and hence Lebesgue nabla integral) on $\Lambda^{n}$ by replacing the family $\mathscr{F}$ of parallelepipeds of the form (3.9) by the family $\mathscr{F}^{\prime}$ of parallelepipeds of the form

$$
V^{\prime}=\left(a_{1}, b_{1}\right] \times \cdots \times\left(a_{n}, b_{n}\right],
$$

with the same volume formula

$$
m\left(V^{\prime}\right)=\prod_{i=1}^{n}\left(b_{i}-a_{i}\right) .
$$

Similarly we can define mixed types of Lebesgue measures on $\Lambda^{n}$ by taking the families of parallelepipeds in which one part of the constituent intervals of the parallelepipeds is left closed and right open and the other part is left open and right closed (see also Remark 2.5).

\section{References}

[1] G. Aslim and G. Sh. Guseinov, Weak semirings, w-semirings, and measures, Bulletin of the Allahabad Mathematical Society 14 (1999), 1-20.

[2] M. Bohner and G. Sh. Guseinov, Partial differentiation on time scales, Dynamic Systems and Applications 13 (2004), no. 3-4, 351-379.

[3] _ Multiple integration on time scales, Dynamic Systems and Applications 14 (2005), no. 34, 579-606.

[4] M. Bohner and A. Peterson, Dynamic Equations on Time Scales, Birkhäuser Boston, Massachusetts, 2001.

[5] M. Bohner and A. Peterson (eds.), Advances in Dynamic Equations on Time Scales, Birkhäuser Boston, Massachusetts, 2003.

[6] D. L. Cohn, Measure Theory, Birkhäuser Boston, Massachusetts, 1980.

[7] P. R. Halmos, Measure Theory, D. Van Nostrand, New York, 1950.

[8] A. N. Kolmogorov and S. V. Fomīn, Introductory Real Analysis, Dover, New York, 1975.

Martin Bohner: Department of Mathematics and Statistics, University of Missouri-Rolla, Rolla, MO 65401, USA

E-mail address: bohner@umr.edu

Gusein Sh. Guseinov: Department of Mathematics, Atilim University, 06836 Incek, Ankara, Turkey E-mail address: guseinov@atilim.edu.tr 